\title{
EGCG prevents the loss of pontine noradrenergic neurons induced by diabetes: a role in diabetic neuropathic pain
}

\author{
Carla Morgado****, João Silva****, André Miranda****, Patrícia Pereira-Terra***, Diogo Raposo* \\ and Isaura Tavares**** \\ * Department of Experimental Biology, Faculty of Medicine of Universiy of Porto, Alameda Professor \\ Hernâni Monteiro, 4200-319 Porto \\ ** IBMC, Rua do Campo Alegre, 823, 4150-180 Porto \\ *** ECS, University of Minho, Campus Gualtar, 4710-057 Braga
}

Diabetes is a major health problem with an alarming increasing prevalence, and is the most frequent cause of neuropathy worldwide. Neuropathy affects $50-60 \%$ of diabetic patients, being a major lifequality impairment for a quarter of these patients [1]. Diabetic neuropathic pain (DNP) is characterized by spontaneous pain, mechanical hyperalgesia and tactile allodynia [1] and is accompanied by functional and neurochemical changes at the peripheral nerves, spinal cord and supraspinal pain control areas [2-8]. Regarding the effects of diabetic neuropathy in the central somatossensory system, it was shown that streptozotocin (STZ)-diabetic rats present spontaneous hyperactivity and hyperexcitability of spinal nociceptive neurons [3, 7], which may be subserving the exacerbated pain responses. The spinal functional changes and pain may be due to increased peripheral input(2), changes in spinal nociceptive modulatory mechanisms [4, 5] and altered supraspinal descending pain modulation [6-8]. Noradrenergic descending pain modulation seems to be impaired since STZ-diabetic rats present decreased numbers of noradrenergic neurons at the A5 and A7 pontine cell groups, along with lower levels of noradrenaline at the spinal cord and higher behavioral responses to pain [8]. This is consistent with the strong noradrenergic projection from A5 and A7 neurons to the spinal dorsal horn and the modulation of nociceptive transmission by local release of noradrenaline [9]. The mechanisms underlying the decrease in noradrenergic neurons in the brainstem during diabetes remain unclear. Our recent findings that diabetes induces oxidative stress damage in neurons from those areas [10], lead us to hypothesize that it may contribute to their loss. Thereafter, with the present study we aimed to evaluate the effects of Epigallocathechin Gallate (EGCG), a potent antioxidant present in green tea, on spinal noradrenaline levels, on A5 and A7 noradrenergic neurons and on behavioral pain responses of STZ-diabetic rats.

Diabetes was induced in male Wistar rats by intraperitoneal injection of STZ (60 mg/kg body weight). Control animals received the vehicle solution. A group of STZ rats received EGCG (2g/l; STZ+EGCG) in drinking water while the other experimental groups received only water (STZ+water and Control). The treatment begun 3 days post-injection and was maintained during 10 weeks. The behavioral pain responses were evaluated before treatment onset and after its completion, using the paw pressure test and the electronic von Frey to determine mechanical hyperalgesia and tactile allodynia, respectively. After the conclusion of the treatment protocol, animals were sacrificed by vascular perfusion (immunohistochemistry study) or decapitation (for ELISA). PFA-fixed brainstems were removed and $40 \mu \mathrm{m}$ thick sections were obtained using a freezing microtome. The pontine noradrenergic neurons were identified in the brainstem sections by immunodetection of tyrosine hydroxylase (TH), an enzyme involved in noradrenaline synthesis, using the avidin-biotin-peroxidade method. Immunorreactive neurons (TH-IR) were visualized using a light microscope and quantified in the A5 and A7 in all experimental groups. Images were captured using a high resolution digital camera coupled to the microscope. Furthermore, freshly removed spinal lumbar enlargements were used to evaluate the levels of noradrenaline by ELISA.

STZ rats developed hyperglycemia, which was maintained until the end of the experiments and was not affected by the EGCG treatment. Mechanical hyperalgesia and tactile allodynia detected in untreated STZ rats were ameliorated by EGCG treatment. The EGCG treatment prevented the 
reduction in TH-IR neurons detected in the A5 and A7 of untreated STZ rats (Figure 1) and normalized the levels of noradrenaline in the spinal cord of STZ-diabetic rats.

This study demonstrates that the antioxidant treatment with EGCG prevents the reduction of noradrenergic neurons in the A5 and A7 and the decrease in spinal noradrenaline content during diabetes, suggesting that the neuronal loss may be mediated by oxidative stress. The improvement of behavioral nociceptive responses indicates that an increased antioxidant intake should be considered as a promising strategy to prevent neurodegeneration at noradrenergic pain control centers of the brain and to ameliorate the altered pain sensations associated to diabetic neuropathy.

\section{References}

1. Tesfaye S et al., Diabetes Care 33: 2285-2293, 2010

2. Obrosova IG. Biochim Biophys Acta 1792: 931-940, 2009

3. Morgado C and Tavares I. Diabetes Metab Res Rev 23: 644-652, 2007

4. Morgado C et al., Neurosci Lett 438: 102-106, 2008

5. Morgado C et al., Diabetes Obes Metab 13:150-159, 2011

6. Morgado C et al., Eur J Pain 14: 693-699, 2010

7. Morgado C et al., Neurobiol Dis 43: 275-284, 2011

8. Morgado C et al., Eur J Pain 13 (supl 1): S167, 2009

9. Pertovaara A, Prog Neurobiol 80: 53-83, 2006

10. Morgado C et al., Diabetologia, 53 (suppl 1): S449-S450
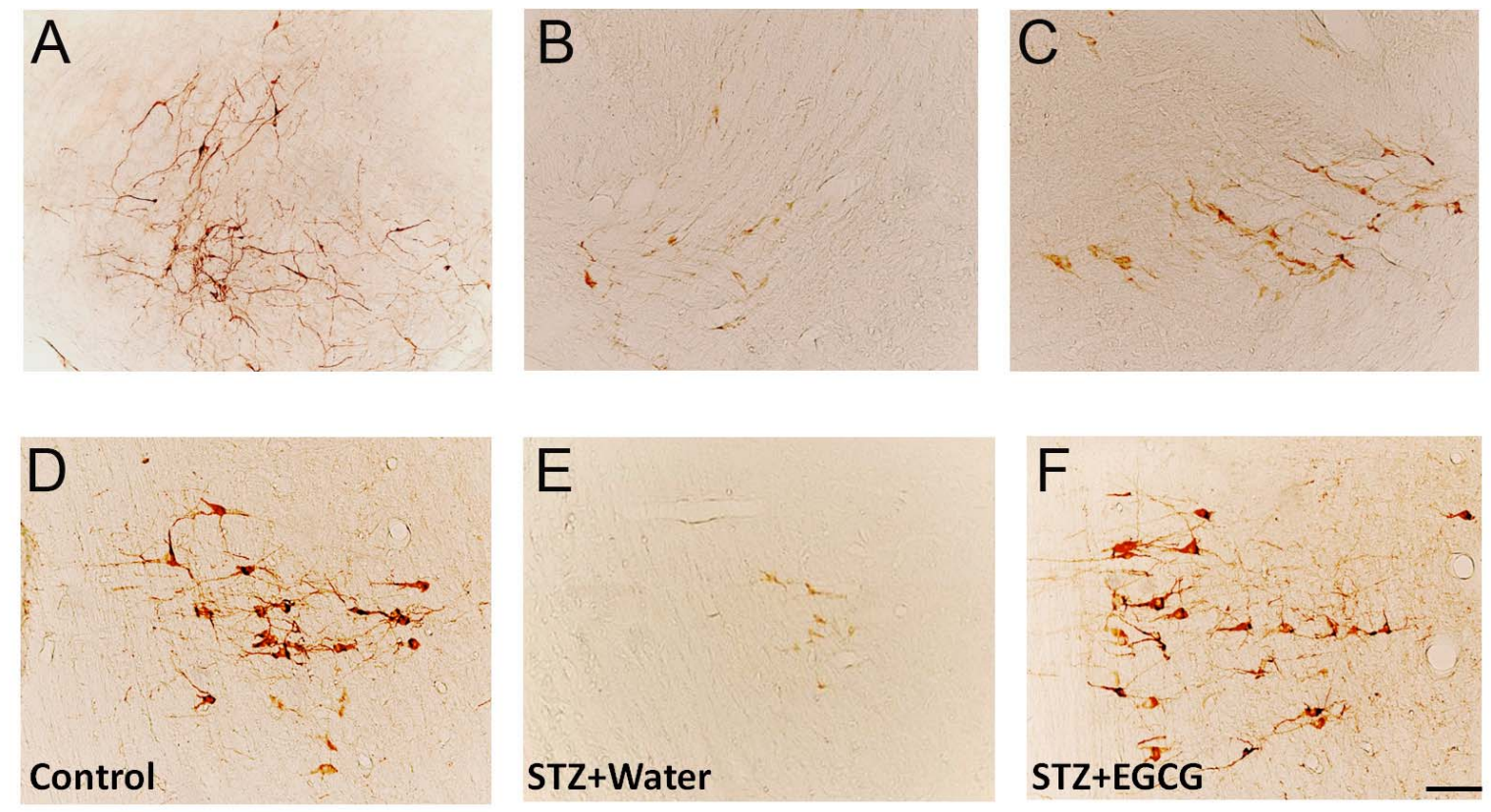

Figure 1. Representative photomicrographs of the noradrenergic A5 (panels A-C) and A7 (D-F) pontine cell groups of control rats (A, D) and STZ-diabetic rats receiving water (B, E) or EGCG (C, E). Scale bar: $100 \mu \mathrm{m}$ (all images at the same magnification).

Supported by IJUP/ UNICER project 